\title{
PESSOAS VULNERÁVEIS E AS PESQUISAS EM SEGURANÇA CONTRA INCÊNDIO
}

\author{
Eriberto Carlos Mendes da Silva ${ }^{1}$ \\ Edna Moura Pinto ${ }^{2}$
}

\section{RESUMO}

O comportamento humano é parte dos componentes que influenciam na evacução de edificações. No caso de pessoas vulneráveis, esta influência ganha pontencialidade pela particularidade associada a mobilidade destas. Este artigo contém o levantamento de pesquisas envolvendo segurança contra incêndio e pessoas vulneráveis, analisando as metodologias recorrentes e sua cronologia, os objetivos, local de realização e os autores frequentes. Para a coleta de informações foram consultados sites, periódicos e bases de dados utilizando palavras chaves relacionadas aos temas segurança contra incêndio e acessibilidade incluindo emergência com pessoas vulneráveis. Os resultados demostram destaque para a simulação computacional e simulados, bem como a velocidade de caminhada enquanto objeto mais pesquisado. Os países da América do Norte e Europa, USA e UK, são apontados como os maiores desenvolvedores de pesquisa e por fim, foi possível constatar que as análises realizadas nas pesquisas tratam das pessoas vulneráveis de forma individualizada em relação às outras e entre si.

Palavras-chave: Acessibilidade. Evacuação. Emergência. Comportamento humano.

\footnotetext{
${ }^{1}$ Doutorando e mestre em Arquitetura e Urbanismo, Universidade Federal do Rio Grande do Norte. Engenheiro Civil com Especialização em Segurança do Trabalho - UFRN - 59078-970 Natal. Telef.: +55 0843215 3776, E-mail: eriberto@ufersa.edu.br

${ }^{2}$ Orientadora, Universidade Federal do Rio Grande do Norte, Arquiteta (UNESP-Bauru) com mestrado em Tecnologia do Ambiente Construído pela Universidade de São Paulo (IAU) e Doutora em Ciências e Engenharia de Materiais pela Universidade de São Paulo (IQSC-IFSCEESC/USP).
} 


\title{
VULNERABLE PEOPLE AND RESEARCHES ON FIRE SAFETY
}

\begin{abstract}
Human behavior is part of the components that influence the evacuation of buildings. In the case of vulnerable people, this influence gains potentiality due to their particular mobility. This article brings a survey of the research developed involving fire safety and vulnerable people, analyzing the most used methodology and its chronology, the objectives, place of accomplishment and the most present authors. The searches were carried out on websites, journals and databases using keywords related to fire safety and accessibility issues including emergency with vulnerable people. The results show that computer simulation and simulated standout. As well as walking speed being the most researched object. The countries in North America and Europe, USA and UK, appear as the greatest research contributors and finally, it was possible to identify that the analyses conducted consider vulnerable people individually in relation to each other and themselves.
\end{abstract}

Keywords: Accessibility, Evacuation, Emergency, Human Behavior.

\section{INTRODUÇÃO}

No desenvolvimento de pesquisas que envolvem evacuação de edificações em situação de incêndio são observadas: as suas condições físicas e sistemas de combate a incêndio (tipos, características, dimensões); as consequências ambientais do incêndio; o tempo de abandono e seus componentes, e ainda o comportamento humano relacionado a estes (PROULX; YUNG, 1996). Entre os métodos aplicados nas pesquisas podemos citar: simulados; experimentos de campo; uso de softwares (simulações 
computacionais); e análise de dados de investigação de sinistros, ou coletados por meio de relatos de sobreviventes, socorristas, equipes de salvamento e testemunhas. As variáveis que fazem os incêndios diferentes entre si interferem e corroboram para dificultar análises e a aplicação dos resultados. Os componentes da reação humana frente a um incêndio e suas consequências como: fumaça, calor, luminosidade e visibilidade, ampliam essa dificuldade. Nesse contexto a inserção de pessoas vulneráveis, consideradas aqui como as que possuem redução ou especificidade com relação à mobilidade (Pessoas com deficiência, com mobilidade reduzida, idosos, gestantes, crianças), se apresenta como um desafio a ser vencido.

As pesquisas sobre abandono de edificações em situações de incêndio incluindo vulneráveis seguem a evolução mundial da garantia dos direitos de inclusão e acessibilidade (PROULX; YUNG, 1996). A acessibilidade física e a garantia de condições igualitárias de abandono às edificações são essenciais para essas pessoas. Assim, os primeiros resultados de estudos contendo pessoas vulneráveis e que buscavam como objetivo melhoria da acessibilidade nas edificações e na área urbanística contribuiu de forma importante para o avanço das pesquisas de abandono de edificações. Estas pesquisas apresentam, por exemplo: valores de velocidade de caminhamento; percepção de riscos e tomadas de decisão; dimensões necessárias para circulação; e, sobretudo a percepção e o comportamento destas diante de obstáculos. Notase que os resultados de uma pesquisa servem de dados e parâmetros para novas, proporcionando avanços, sobretudo quando se leva em conta a dificuldade de coleta de dados empíricos e necessidade de definir padrões com maior nível de generalização (CHRISTENSEN et al., 2014). Os ajustes de regulamentos de segurança contra incêndio e consequêntemente o surgimento de propostas de alterações das características das edificações decorrem de estudos que, em grande parte, advém de investigação e análise de sinistros. $O$ caso do WTC (Wold Trade Center) nos Estados Unidos e o da Boate Kiss no Brasil são exemplos disto. Entretanto, a legislação de inclusão e acessibilidade 
evolue em ritmo maior e independente de sinistros. Por sua vez, o volume de pesquisas que envolvem pessoas vulneráveis e situações de evacuação de edificações ainda necessita de incremento para acompanhar essa evolução. As pesquisas com maior frequência têm como alvo análise de situações com pessoas vulneráveis individualizadas. Constata-se a necessidade de mais estudos que envolvam a interação dos diversos tipos de pessoas com deficiência ou necessidade especial de locomoção entre si, e com as demais.

\section{OBJETIVO}

O presente artigo busca apresentar uma análise de pesquisas envolvendo pessoas vulneráveis que se relacionam com situações com evacuação de edificações, observando suas características e abordagem.

\section{METODOLOGIA}

O estudo é desenvolvido com uma análise qualitativa, exploratória da produção científica depositada em bancos de dados nacionais e internacionais de acesso livre, sites de busca como Google scholar, periódicos, anais de congressos e simpósios internacionais, entre outros. Nas buscas utilizou-se palavras chaves relacionadas aos temas de acessibilidade e segurança contra incêndio. As palavras e termos utilizados na pesquisa foram: accessibility, handicapped, Individual with disability, reduced mobility, disability, impairment, wheelchair, elderly, child, emergency, evacuation, fire, simulation, simulated. Fazendo uso da língua pátria do autor foi feita pesquisa com as referidas palavras e termos em português do Brasil. Tomou-se como base inicial do estudo artigos de revisão de literatura e suas citações. Após a primeira seleção dos documentos foram utilizados filtros de refinamento para comprovar a interação dos assuntos (palavras chaves e sinônimos). A análise dos documentos se fez por meio da identificação do autor, ano e país da realização 
da pesquisa, tipo de ambiente ou edificação da realização da pesquisa, da população alvo, do objetivo, da interação entre os participantes e, do(s) método(s) empregado(s).

\section{DESENVOLVIMENTO E RESULTADOS}

O desenvolvimento do estudo tomou como base inicial a revisão de literatura realisada por Christensen et al. (2014), Sharifi et al. (2015) e Hamilton, Lennon e O'raw (2017), acrescentados de 120 documentos pesquisados conforme metodologia descrita.

Neste estudo além dos dados de identificação dos documentos pesquisados como autor, ano de publicação/pesquisa, país da pesquisa, tipo de deficiência ou condição limitante de locomoção da população analisada, o objetivo, foi observada a metodologia utilizada para alcançá-la e ainda se a pesquisa trabalhou com dados de interação entre as diversas pessoas envolvidas. No caso da interação, tomou-se como parâmetro a observação de resultados que levaram em conta a influência dos participantes entre si. Para a identificação da metodologia denominou-se:

A. Simulado: evento realizado em ambiente real, sem modificações; Experimento de campo;

B. Simulação computacional: utilização de software de simulação de evacuação/fluxo, desempenho de equipamentos, instalações ou projetos;

C. Revisão de literatura: busca documental em bases de dados científicos e acadêmicos, revistas, sites, congressos e simpósios especializados nos temas;

D. Aplicação de Questionários, entrevistas: busca de informações direcionadas à população alvo através de instrumentos elaborados para tal; 
E. Análise de dados: resumo das ações de coleta de dados de participantes de eventos reais, análises comparativas de pesquisas anteriores, observações, check list.

Iniciando o desenvolvimento deste estudo vimos que em seu artigo de revisão de 2014, Christensen et al. (2014) obtiveram como conclusão que há poucos estudos acadêmicos que enfoquem o ambiente construído e a influência deste na capacidade de pessoas com deficiência em situações de emergência. Constatam ainda que a maioria dos estudos se concentra nas características funcionais específicas de cada pessoa com deficiência na interação com o ambiente construido, prevalescendo nestas a velocidade de caminhamento. Para os autores, as demais literaturas pesquisadas apresentavam uma descrição das pessoas com deficiência com sendo um obstáculo no ambiente construido ou a interpretação destas sobre as condições de uso. Indicam como necessidade nas simulações computacionais, uma base de dados empíricos mais aprofundados. Na revisão temos que as pesquisas tratam, na sua maioria, os diversos tipos de pessoas com deficiências de forma separada, com poucos estudos relatando o comportamento dessas pessoas compondo uma população heterogêna com as pessoas sem deficiência ou, de um tipo de pessoa com deficiência em relação a outro.

Os autores do artigo de revisão, Sharifi et al. (2015), tiveram como objetivo "examinar os impactos das pessoas com deficiência na velocidade de marcha da multidão e estudar os impactos de diferentes instalações de circulação interna como escadas, rampas, corredores e circulações, nos movimentos de vários grupos de pedestres". Neste estudo as análises contemplam populações heterogêneas e homogêneas em situações de comparação quanto ao desempenho em ambientes laboratoriais criados para análise de comportamento e influência mútua em eventos de evacuação simulados. Sharifi et al. (2015) citam a revisão de Christensen et al. (2014) e corroboram com suas conclusões. Acrescentam novos estudos aos já citados 
por Christensen et al. (2014), relacionando as pesquisas que servem de base de dados para um grande número de outras como é o caso de Clark-Carter et al. (1986), Rubadiri et al. (1997), Boyce et al. (1999 a,b,c), Wright et al. (1999).

No estudo realizado por Hamilton, Lennon e O'raw (2017), encontra-se uma revisão de literatura recente quanto a crianças em situações de emergência. Neste caso os autores chegam à relação de diminuição do tempo de pré-movimento e evacuação com o aumento da idade das crianças e da quantidade de repetição dos simulados de evacuação. Ainda comentam sobre influência da idade no comportamento e da diferença de densidade provocada pelas dimensões corporais de crianças menores do que de adultos.

Das pesquisas selecionadas nas buscas analisadas neste trabalho encontramos pesquisas que não fazem relação direta entre a acessibilidade ou com a segurança contra incêndio, nem mesmo quando abordavam dimensões de acessos, circulações, sinalização, por exemplo. Estas representaram 38\% do total pesquisado e foram descartadas.

No desenvolvimento do estudo foi possível notar que os simulados e as pesquisas de revisão de literatura na discussão de temas relacionados foram os métodos recorrentes. Por meio da Tabela 1 e complementarmente pela Figura 1, podemos identificar os principais métodos adotados.

Tabela1 - Método de pesquisa (Acessibilidade e segurança contra incêndio)

\begin{tabular}{ccc}
\hline Método & Quantidade & Percentual \\
\hline Revisão de literatura & 19 & $27 \%$ \\
Simulação computacional & 5 & $7 \%$ \\
Simulado & 18 & $26 \%$ \\
Simulado e simulação & 3 & $4 \%$ \\
computacional & 1 & $1,4 \%$ \\
Simulado e entrevista & 1 & $1,4 \%$ \\
Simulado e questionário & 6 & $9 \%$ \\
Questionário e entrevistas & 5 & $7,2 \%$ \\
Misto & 11 & $16 \%$ \\
\hline Outros & Fonte: Elaborada pelo autor. & \\
\hline
\end{tabular}

Fonte: Elaborada pelo autor. 
As revisões de literatura aparecem na discussão de temas específicos, tal como se faz notar na pesquisa de Gromer (2016) que relata sobre modelo de decisão de movimento de ocupantes a evacuação de emergência para pessoas com deficiência na pesquisa de Hashemi (2018) e as mudanças demográficas e a variação da mobilidade na pesquisa de Boyce (2017).

Os simulados e as simulações computacionais foram os métodos mais utilizados levando em conta a soma do uso individualizado (Hunt; Galea; Lawrence, 2015), (SORENSEN; DEDERICHS, 2015), (FAN et al., 2019), (KULIGOWSKI et al., 2013), (LOURENÇO, 2013), (VALENTIN; ONO, 2014), (KESLER et al., 2017), e em conjunto com outros métodos como entrevistas (BOYCE et al., 2017) e questionários (SHIELDS; BOYCE, 2000).

Figura 1 - Distribuição da Metodologia das Pesquisas

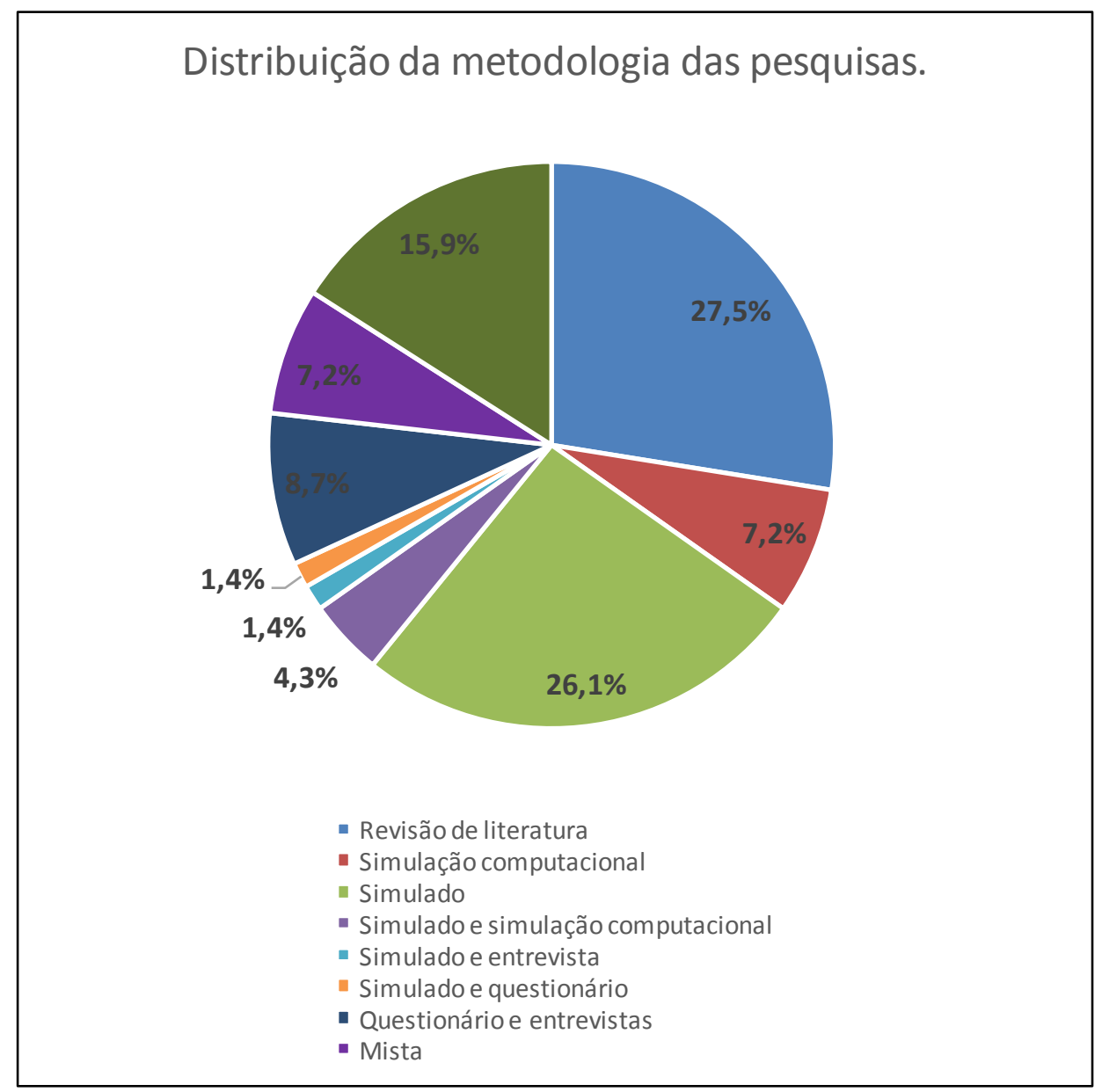


$\mathrm{Na}$ análise do avanço cronológico das pesquisas, por meio do ano da publicação, temos que a partir do ano de 2011 os simulados e as simulações computacionais aparecem com maior frequência, somando $65 \%$ e $75 \%$ respectivamente, conforme mostra a Quadro 1.

Quadro 1 - Cronologia de pesquisas relacionadas com simulação e simulado

\begin{tabular}{c|c|c}
\hline \multicolumn{2}{c}{ DISTRIBUIÇÃO DE QUANTIDADE DE PESQUISAS POR PERÍODO, EM ANOS } \\
\cline { 2 - 3 } Ano & Simulado & Metodologia (quantidade) \\
\hline $1984|--| 2000$ & 5 & 1 \\
\hline $2000--\mid 2010$ & 3 & 6 \\
\hline $2010--\mid 2019$ & 15 & $\mathbf{8}$ \\
\hline Total & $\mathbf{2 3}$ & $75 \%$ \\
\hline $\begin{array}{c}\text { Porcentagem de } \\
\text { publicações após 2011. }\end{array}$ & $65 \%$ & \\
\hline
\end{tabular}

Fonte: próprio autor por meio dos dados da presente pesquisa, 2019.

Os dados indicam os caminhos tomados para as pesquisas onde, a necessidade de dados empíricos é imprescindível para alimentação dos sistemas computacionais baseados em agentes. A Figura 2 mostra um gráfico que representa o aumento da aplicação das duas metodologias na última década. 
Figura 2 - Gráfico com tendências cronológica de pesquisas relacionadas com simulação e simulado

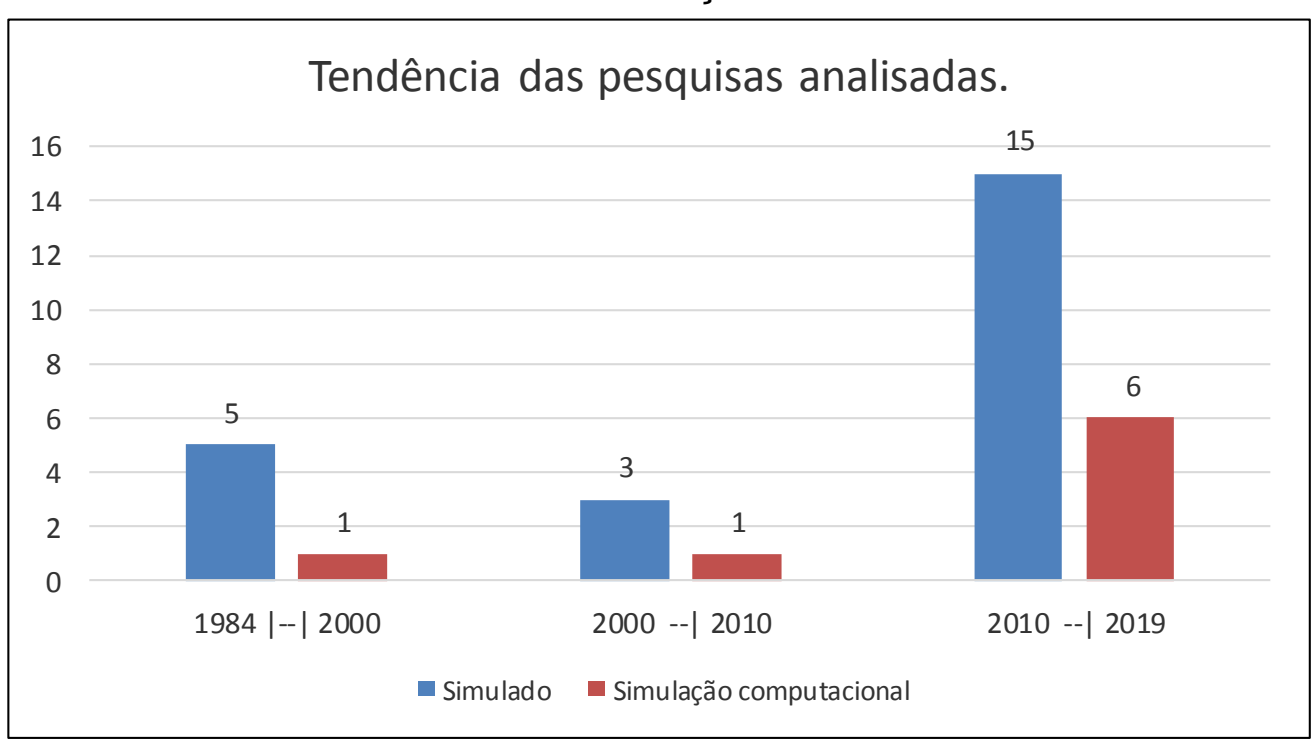

Fonte: próprio autor, elaborado através dos dados da presente pesquisa, 2019.

Ainda, corroborando com esse pensamento, existe a necessidade de fornecer dados sobre o desempenho de sistemas de evacuação com população heterogênea para subsidiar projetos mais eficientes.

Estados Unidos e o Reino Unido lideram a lista de países que mais produzem pesquisas sobre o tema de acessibilidade com segurança contra incêndio contabilizando neste estudo $29 \%$ e $17 \%$, respectivamente. O restante da Europa, no seu conjunto, aparece com pouco mais de 20\%. A Ásia 12\%, representada pela China, Japão e Irã. O Brasil aparece na pesquisa devido a busca no idioma português, no entanto, o alcance é dificultado justamente por este fator. Ainda temos Austrália, Nova Zelândia, Nigéria, entre outros, aparecendo de forma discreta. A Figura 3, mostra no mapa mundial uma representação da distribuição das pesquisas por local de publicação, continentes e países. 
Figura 3 - Distribuição geográfica das pesquisas analisadas.

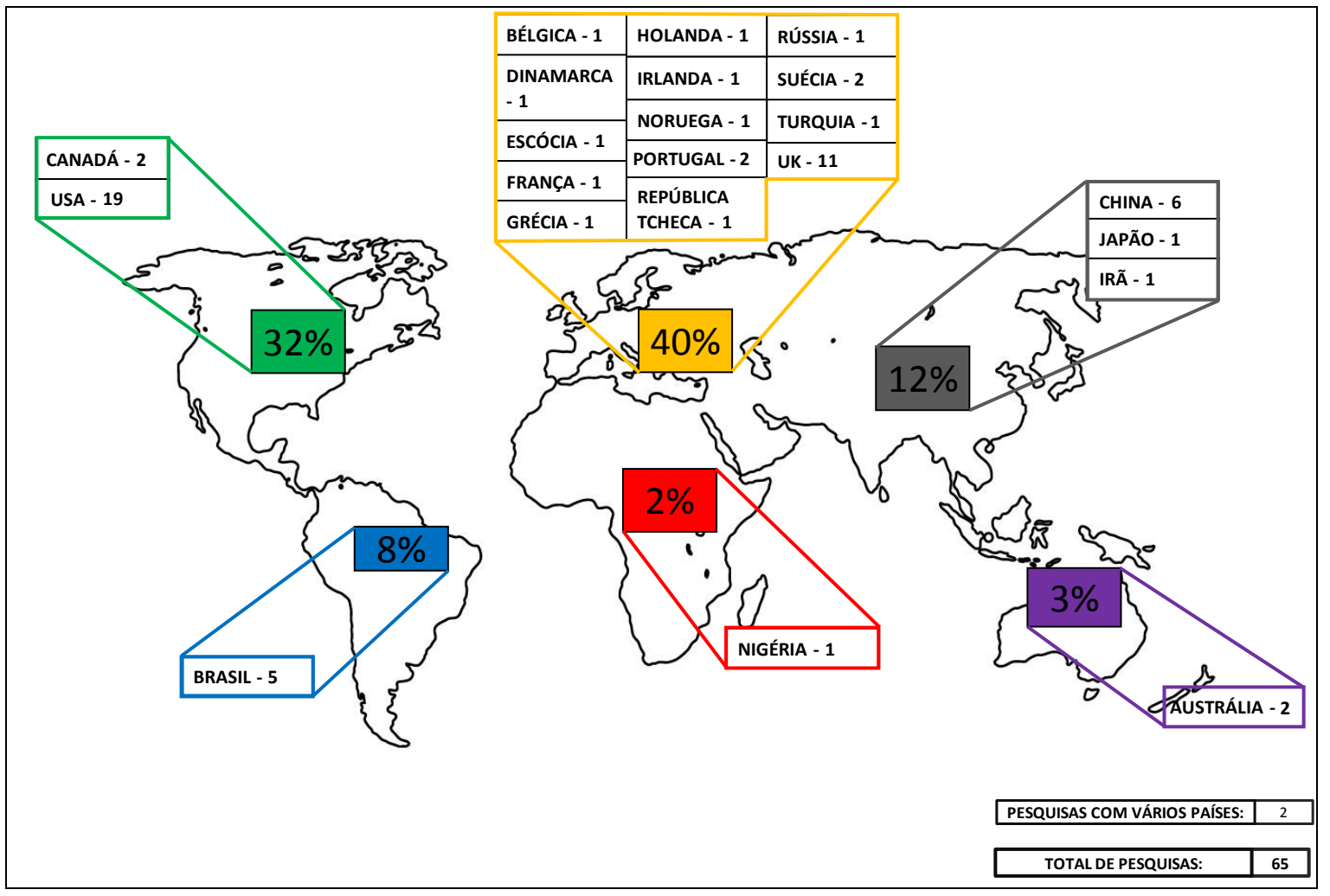

Fonte: Elaborada pelo autor (2019).

Dentre os pesquisadores que mais se destacam encontramos Karen Boyce, Richard D. Peacock, Erica Kuligowski, Keith Christensen, T. J. Shields, G. Proulx, G. W. H. Silcock, entre outros.

Os locais mais pesquisados são ambientes internos de edifícios em geral, incluindo os públicos e comerciais, principalmente edifícios altos com foco na evacuação vertical (escadas, elevadores de emergência) (LAVENDER et al., 2014), (RONCHI; NILSSON, 2013), (SHIELDS; BOYCE; MCCONNELL, 2009), (SPEARPOINT; MACLENNAN, 2012). Seguem, especificamente quanto a crianças (NAJMANOVÁ; RONCHI, 2017), (FAN et al., 2019), (LOURENÇO, 2013), as escolas e quanto aos idosos (VALENTIN; ONO, 2014), (FAHY, 2015), as casas de repouso e locais de assistência de saúde. 
Como objetivos buscados nas pesquisas foram contemplados: a velocidade de caminhada, velocidade de evacuação, tempo de pré-movimento e de evacuação, e estratégias de evacuação, somando 50\% do total. Exemplo destes são as pesquisas de Hamilton, Lennon e O'raw (2017); Hashemi (2018); Hunt, Galea e Lawrence (2015); Sorensen e Dederichs (2015); Valentin e Ono (2014); Boyce et al (2017); Shields, Boyce e Mcconnell (2009); Fahy (2015). O comportamento humano é citado em análises de tempo de pré-movimento por Hamilton, Lennon e O'raw (2017), tomadas de decisões por Gromer (2016), impactos no desenvolvimento da evacuação (congestionamento) por Chen et al (2018), percepção de riscos. Essas ainda se relacionam com pesquisas que trabalham com medidas de velocidade de pré-movimento, por exemplo.

Para a população utilizada nas pesquisas temos as pessoas com deficiências representadas na maior parte por pessoas com mobilidade reduzida, principalmente tomando as pessoas usuárias de cadeiras de rodas como parâmetro. Idosos, crianças, obesos e deficientes auditivos e visuais também aparecem de forma específica. As pesquisas tratam as pessoas vulneráveis de forma individualizada nas avaliações e análises, como nos estudos de Hamilton, Lennon e O'raw (2017); Hunt, Galea e Lawrence (2015); Sorensen e Dederichs (2015); Kuligowski et al. (2013). As informações de interação entre essa população de pessoas com deficiências entre si ou com outros participantes aparecem nas pesquisas de forma minoritária com 36\% das pesquisas. Entre essas observa-se que a interação relatada consiste em informações de congestionamento nas rotas de fuga, necessidade de auxílio para deslocamento, depoimentos de sentimento de insegurança, medo ou desconfiança na eficácia da estratégia de evacuação.

\section{CONCLUSÕES}

$\mathrm{Na}$ revisão em tela tem-se como resultado uma maior frequência de pesquisas sobre a velocidade de caminhada de pessoas vulneráveis, seguidas 
por aquelas que relatam interação na espacialidade dos ambientes, sua percepção e comportamento diante dos obstáculos. A grande maioria refere-se aos deficientes ou pessoas com mobilidade reduzida, individualmente. Em conclusão, existe uma lacuna nas pesquisas que buscam informações sobre o comportamento de interação de pessoas vulneráveis nos seus diversos tipos em situações de emergência, como a influência da ação de uma sobre a outra, e ainda as alternativas vislumbradas por estes para solucionar a questão. Desta forma é necessário ampliar a gama de pesquisadores embuídos em discutir a relação entre as pessoas vulneráveis, prevendo a garantia de coexistência segura nos ambientes de convívio social. Novas pesquisas neste rumo tenderão a atender, de forma mais precisa, às necessidade de projetistas e das próprias pessoas vulneráveis nas situações de emergências.

\section{AGRADECIMENTOS}

Ao Programa de Pós Graduação em Arquitetura e Urbanismo da Universidade Federal do Rio Grande do Norte pelo apoio no desenvolvimento de pesquisas sobre o tema de segurança contra incêndio.

Ao IGNIS - Grupo de Estudos Sobre Segurança Contra Incêndio UFRN.

\section{REFERÊNCIAS}

BOYCE, K. Safe evacuation for all-Fact or Fantasy? Past experiences, current understanding and future challenges. Fire Safety Journal, v. 91, p. 28-40, 2017.

BOYCE, M. W. et al. Design of instructions for evacuating disabled adults. Applied ergonomics, v. 58, p. 48-58, 2017. 
CHEN, L. et al. Elementary students' evacuation route choice in a classroom: A questionnaire-based method. Physica A: Statistical Mechanics and its Applications, v. 492, p. 1066-1074, 2018.

CHRISTENSEN, K. M. et al. The relationship between the design of the built environment and the ability to egress of individuals with disabilities. Review of Disability Studies: An International Journal, v. 2, n. 3, p. 24-35, 2014.

FAHY, R. Panel: life safety options for people with disabilities-how far have we come?. Fire and Materials, v. 39, n. 4, p. 475-485, 2015.

FANG, Z. M. et al. Experimental study on the movement characteristics of 5-6 years old Chinese children when egressing from a pre-school building. Safety science, v. 113, p. 264-275, 2019.

GRONER, N. E. A decision model for recommending which building occupants should move where during fire emergencies. Fire Safety Journal, v. 80, p. 2029, 2016.

HAMILTON, G. N.; LENNON, P. F.; O'RAW, J. Human behaviour during evacuation of primary schools: Investigations on pre-evacuation times, movement on stairways and movement on the horizontal plane. Fire Safety Journal, v. 91, p. 937-946, 2017.

HASHEMI, M. Emergency evacuation of people with disabilities: A survey of drills, simulations, and accessibility. Cogent Engineering, v. 5, n. 1, p. 1506304, 2018.

HUNT, A.; GALEA, E. R.; LAWRENCE, Peter J. An analysis and numerical simulation of the performance of trained hospital staff using movement assist devices to evacuate people with reduced mobility. Fire and Materials, v. 39, n. 4, p. 407-429, 2015.

KESLER, R. M. et al. Egress efficacy of persons with multiple sclerosis during simulated evacuations. Fire technology, v. 53, n. 6, p. 2007-2021, 2017.

KULIGOWSKI, E. et al. Stair evacuation of older adults and people with mobility impairments. Fire Safety Journal, v. 62, p. 230-237, 2013.

LAVENDER, S. A. et al. Evaluating the physical demands on firefighters using hand-carried stair descent devices to evacuate mobility-limited occupants from high-rise buildings. Applied ergonomics, v. 45, n. 3, p. 389-397, 2014.

LOURENÇO, A L. S. Evacuação Numa Instituição Particular de Solidariedade Social com Quatro Valências (Lar, Centro de Dia, Creche e 
CATL). 2013. 85 f. Dissertação (Mestrado) - Curso de Engenharia de Segurança e Higiene Ocupacionais, Faculdade de Engenharia da Universidade do Porto, Porto, 2013.

NAJMANOVÁ, H.; RONCHI, E. An experimental data-set on pre-school children evacuation. Fire technology, v. 53, n. 4, p. 1509-1533, 2017.

PROULX, G.; YUNG, D. Evacuation procedures for occupants with disabilities in highrise buildigns. 3rd International Symposium Applications Of Performace, Tel-aviv, p.1-10, 1996.

RONCHI, E.; NILSSON, D. Fire Evacuation in High-Rise Buildings: A Review on Human Behaviour and Modelling Research. Lund University. Department of Fire Safety Engineering and Systems Safety, v. 1, n. 2, p. 1-21, 2013.

SHARIFI, M. S. et al. Analysis of walking speeds involving individuals with disabilities in different indoor walking environments. Journal of Urban Planning and Development, v. 142, n. 1, p. 1-10, 2015.

SHIELDS, T. J.; BOYCE, K. E. A study of evacuation from large retail stores. Fire Safety Journal, v. 35, n. 1, p. 25-49, 2000.

SHIELDS, T. J.; BOYCE, K. E.; MCCONNELL, N. The behaviour and evacuation experiences of WTC 9/11 evacuees with self-designated mobility impairments. Fire Safety Journal, v. 44, n. 6, p. 881-893, 2009.

SORENSEN, J. G.; DEDERICHS, A. S. Evacuation characteristics of visually impaired people-a qualitative and quantitative study. Fire and Materials, v. 39, n. 4, p. 385-395, 2015.

SPEARPOINT, M.; MACLENNAN, H. A. The effect of an ageing and less fit population on the ability of people to egress buildings. Safety science, v. 50, n. 8, p. 1675-1684, 2012.

VALENTIM, M. V.; ONO, R. Qualidade do projeto de saídas de emergência em edificações para pessoas com deficiência. Xv Encontro Nacional de Tecnologia do Ambiente Construído, Maceió, Alagoas-Brasil, p.1943-1952, 11 nov. 2014. 